\title{
Satisfacción en las Relaciones de Pareja en la Adultez Emergente: El Rol del Apego, la Intimidad y la Depresión
}

\section{Satisfaction in Intimate Relationships in Emerging Adulthood: The Role of Attachment, Intimacy and Depression}

\author{
Diana Rivera \\ Constanza Cruz \\ $\mathrm{y}$ \\ Catalina Muñoz \\ Universidad Diego Portales, Chile
}

(Rec: 11 de enero de 2010; Acep: 31 de diciembre de 2010)

\begin{abstract}
Resumen
Esta investigación se propone caracterizar las relaciones de pareja en el período de la adultez emergente, y establecer la relación entre la satisfacción con la relación de pareja y los estilos de apego, la presencia de síntomas depresivos y el miedo a la intimidad. Participaron 120 jóvenes entre 18 y 26 años, quienes contestaron un cuestionario autoadministrado. Los resultados indican que la satisfacción con la relación disminuye por el grado de ansiedad y el miedo a la intimidad. Existen similitudes entre las relaciones amorosas en la adultez emergente y las relaciones adultas en base a su compromiso y duración.
\end{abstract}

Palabras clave: adultez emergente, pareja, apego, intimidad, depresión

\begin{abstract}
This research aims to characterize the relationships in the period of emerging adulthood, and establish the relationship between satisfaction with the relationship and attachment, the presence of depressive symptoms and fear of intimacy. Participants 120 young people, between 18 and 26 who answered a self-administered questionnaire. The results indicate that satisfaction with the relationship falls by the degree of anxiety and fear of intimacy. There are similarities between romantic relationships in emerging adulthood and adult relationships based on their commitment and duration.
\end{abstract}

Key words: emerging adulthood, romantic relationship, attachment, intimacy, depression

Correspondencia: Diana Rivera Ottenberger, Facultad de Psicología, Universidad Diego Portales, Vergara 275, Santiago; fono: (56 2) 676 2579. E-mail: diana.rivera@udp.cl 


\section{Introducción}

\section{La adultez emergente}

En las sociedades industrializadas occidentales se ha producido un paulatino retroceso en la edad en que los jóvenes finalizan sus estudios, dejan el hogar parental, se integran al mercado laboral, se independizan económicamente y consideran conformar una familia (Cohen, Kasen, Chen, Hartmark \& Gordon, 2003; Instituto Nacional de la Juventud [INJU], 2006). Esto ha generado la definición de un nuevo período evolutivo comprendido entre los 18 y 25 años, denominado adultez emergente (Arnett, 2000). En este período, la exploración individual de las posibilidades que la vida proporciona es mayor que en ningún otro período de la vida (Arnett, 1998, 2000, 2001). Últimamente existe un interés creciente en el estudio de la transición entre la adolescencia y la adultez, pues en este período es necesario desarrollar habilidades y competencias propias de este estadio del desarrollo (Cohen et al., 2003; Gaudet, 2007).

El establecimiento de una relación de pareja estable y perdurable en el tiempo es una de las tareas más importantes que los jóvenes deben realizar en la adultez emergente (Arnett, 2001). Esta búsqueda se inicia en la adolescencia y se vincula con la capacidad de establecer relaciones de intimidad (Erickson, 1963). Las relaciones amorosas adolescentes transitan a lo largo de cuatro etapas, siendo las relaciones de pareja comprometidas propias de la finalización del período (Connolly \& Goldberg, 1999). Estas se caracterizan por ser más largas, incluyen la atracción sexual, la intimidad e integran otros elementos, como la capacidad de proporcionar cuidados al otro. Shulman y Scharf (2000) refieren que en esta última etapa, las relaciones amorosas están orientadas a la búsqueda de mayor cercanía emocional y cuidado hacia el otro, comenzando a asemejarse a las relaciones de pareja adultas.

Las relaciones amorosas en la adultez emergente presentan múltiples alternativas y denominaciones, dependiendo de niveles diferenciales de involucramiento emocional, exclusividad, intimidad sexual, permanencia en la relación y su formalización (Shulman \& Seiffge-Krenke, 2001). Esta relaciones se dan en un contexto social donde coexisten diversas concepciones del amor, del sexo, del placer y legitimidad de diversas formas vinculares (Ferrer, Bosch, Navarro, Ramis \& García, 2008). En Chile, una posibilidad relacional son los encuentros esporádicos o casuales con diversos grados de intimidad sexual, sin compromiso futuro, que pueden establecerse con una o varias personas. Estas serían las relaciones más inestables y casuales. También pueden darse relaciones de amistad que ocasionalmente involucran diversos grados de intimidad sexual sin que las personas se reconozcan en una relación de pareja (amigos con ventaja). Otra modalidad refiere a que, seguido a una atracción inicial, existe el interés por compartir y conocerse mutuamente, sin que esto conlleve ningún compromiso particular (salir o andar con alguien). Esta primera relación de atracción y conocimiento puede derivar en una relación de mayor compromiso, afecto, intimidad y formalidad (pololeo), es decir, una relación de pareja más seria, que puede derivar a otro tipo de relación, o bien finalizar. En caso que esta relación perdure y se profundice, puede derivar en una relación que considera un compromiso matrimonial futuro (noviazgo).

La diversidad de nomenclaturas que asumen las relaciones de pareja genera problemas en la definición del objeto de estudio, pues en distintas culturas su denominación y significado puede ser diferente (Sánchez, Ortega, Ortega \& Viejo, 2008).

El tipo de relación varía según la inserción del joven en el sistema educativo y laboral. En el caso chileno, los jóvenes que se dedican sólo a estudiar se encuentran mayoritariamente sin pareja (57.4\%), y los que estudian y trabajan, en un $41.9 \%$ establecen una relación de mayor compromiso y perdurabilidad (pololeo). A diferencia de los casos anteriores, los jóvenes unidos en vínculo de matrimonio en su mayoría se encuentran insertos en el mercado laboral (INJU, 2006). A su vez, el tipo de vínculo varía con la edad, siendo las relaciones de pareja de menor compromiso más frecuentes entre los jóvenes de 15 a 19 años. En el tramo de 20 a 24 años, la relación de pololeo es la relación más frecuente (33\%), mientras que entre los 25 a 29 años el vivir en pareja (39.2\%) es la relación mayoritaria (INJU, 2006).

\section{Satisfacción con la relación, apego, intimidad y depresión}

Los jóvenes difieren en su capacidad de encontrar pareja y establecer una relación. Uno de los factores que puede afectar este proceso, refiere a las experiencias que se ha tenido con el cuidador primario -habitualmente la madre- en cuanto la relación de apego. Esta relación primaria establecería un prototipo de relación a través de la conformación de modelos operativos internos, que se extendería a las relaciones de amistad y pareja, afectando las características y grado de satisfacción con las mismas (Bowlby, 1988; Feeney \& Noller, 1986; Kirkpatrick \& Davis, 1994). Efectivamente, Brumbaugh y Fraley (2006) sostienen que existe una transferencia de los estilos de apego a parejas potenciales imaginarias, aún cuando éstas últimas difieran significativamente en sus atributos de parejas anteriores. Las personas con estilos de apego más ansiosos y evitativos, anticipan sentirse más ansiosos y más evitativos con esa pareja potencial. Esto implica que los modelos operativos internos constituyen una disposición interpersonal que influencia las interacciones iniciales en las relaciones de pareja y el destino de las mismas. Sin embargo, la transferencia de los modelos operativos internos globales a otras relaciones, y la existencia de modelos 
operativos específicos a una relación en particular son un aspecto aún en estudio (Collins, Guichard, Ford \& Feeney, 2004; Collins \& Read, 1994).

Una relación de apego seguro con el cuidador primario promueve una relación de pareja satisfactoria, pues se asocia a esta modalidad vincular una serie de efectos beneficiosos, como la capacidad de intimar, de dar y recibir apoyo, y de proporcionar y solicitar cuidados (Cassidy, 2001; Collins \& Sroufe, 1999). Por el contrario, los estilos de apego inseguros darían lugar a relaciones más conflictivas, menos duraderas y las personas tendrán mayor probabilidad de padecer alteraciones emocionales, como es la depresión (Bartholomew \& Horowitz, 1991; Brennan, Clark \& Shaver, 1998). También los estilos de apego inseguros han sido asociados a una mayor prevalencia de problemas emocionales y patologías psiquiátricas (Dottan, Cohen, Iwaniec, Sapir \& Weizman, 2003; McNally, Palfai, Levine \& Moore, 2003). En los jóvenes, la presencia de síntomas depresivos se asocia a modelos negativos del sí mismo y de los otros, es decir, con estilos de apego inseguros (Dávila, Steinberg, Kachadourian, Cobb \& Fincham, 2004; Kobak, Sudler \& Gamble, 1991; Marchand-Reilly, 2009). Así, la presencia de sintomatología depresiva puede disminuir la probabilidad de establecer relaciones afectivas y de pareja, limitando las experiencias juveniles. Este trastorno afecta al $13.4 \%$ de los jóvenes varones y al $18.1 \%$ de las mujeres entre 17 y 24 años de edad (Ministerio de Salud [MINSAL], 2004).

Un segundo factor estrechamente ligado al apego que influencia el establecimiento y la calidad de la relación de pareja, es la capacidad de establecer una relación de intimidad. Las dificultades en este plano pueden llevar al rechazo y aislamiento, pues durante la adolescencia y la juventud el establecimiento de una relación de pareja no sólo es una fuerte motivación, sino que también constituye una forma de inserción social (Erickson, 1963; Collins, 2003). Así, la intimidad es tanto un logro de esta etapa como también un aspecto importante al momento de establecer una relación de pareja satisfactoria, lo cual se ve favorecido por la existencia de un apego seguro (Bartholomew et al., 1991; Cassidy, 2001; Hazan \& Shaver, 1987).

Descutner y Thelen (1991) definen el miedo a la intimidad como "...la capacidad inhibida de un individuo debido a la ansiedad, de intercambiar pensamientos y sentimientos de relevancia personal con otra persona altamente valorada" (p.219). Así, se establece una relación entre la capacidad de intimar y los estilos de apego. El establecimiento de una relación íntima tiene efectos beneficiosos, como por ejemplo, afrontar de mejor manera el estrés, lo cual es más característico de las mujeres (Taylor, Klein, Lewisa, Gruenewald, Gurungy \& Updegraff, 2000).

El objetivo del estudio fue caracterizar las relaciones de pareja y conocer la relación entre la satisfacción relacional y las variables de ansiedad y evitación (apego), el rol de la dificultad de intimar y la presencia de síntomas depresivos en la adultez emergente.

\section{Método}

\section{Participantes}

Los participantes fueron 120 estudiantes universitarios (63 mujeres y 57 varones). El muestreo fue de tipo no probabilístico e incluyó cuatro carreras de una misma universidad: medicina, psicología, ingeniería comercial y auditoría. El rango de edad fue entre 17 y 26 años ( $M=$ 20.58; $D T=1.96)$.

\section{Procedimiento}

Se aplicó un cuestionario de autorreporte en sala de clases, previo firma del consentimiento por parte de los estudiantes. Se informó acerca de los objetivos del estudio, enfatizándose la voluntariedad de la participación, la confidencialidad y anonimato de la información proporcionada.

\section{Instrumentos}

Los instrumentos empleados fueron adaptados al español hablado en Chile, mediante modificaciones mínimas en la redacción de alguno de sus ítems, por consenso de un panel de dos expertos.

Satisfacción con la relación. Se utilizó la escala Relationship Assessment Scale (RAS) de Hendrick (1988), que evalúa la satisfacción global con la relación. Este instrumento autoadministrado consta de siete ítems evaluados en una escala Likert de 1 a 5, donde 1 corresponde a no me representa para nada y 5 a me representa totalmente. En este estudio la consistencia del instrumento fue según el alpha de Cronbach de .71.

Miedo a la Intimidad. Se utilizó la escala Fear Intimacy Scale (FIS) de Descutner y Thelen (1991), que permite evaluar el miedo a la intimidad en personas que tienen y no tienen una relación de pareja. El instrumento tiene dos partes. La parte A consta de 30 ítems donde se solicita a los respondientes que se imaginen en una relación de pareja e indiquen cuánto les representa las aseveraciones planteadas. La parte B consta de cinco ítems que refieren a evaluar la experiencia en relaciones pasadas. Cada ítem es evaluado en una escala de cinco puntos, en un rango de no es característico de mi persona (1) a es totalmente característico de mi persona (5). Mientras más alto el puntaje, mayor ansiedad en las relaciones cercanas. En este estudio se modificó la instrucción, solicitando en la parte A que las personas que estuviesen en una relación de pareja, contestaran en base a esa relación. Esta modificación se enmarca dentro de las sugerencias realizadas por Descutner et al., quienes refieren la necesidad de investigar el miedo a la intimidad en distintas relaciones, como por ejemplo, en la relación amorosa actual, en relaciones familiares, en la relación terapéutica, entre otras. La consistencia interna de la escala medida a través del alfa de Cronbach fue de .87. 
Evaluación del Apego. El instrumento utilizado fue Experience in Close Relationships Revised $(E C R-R)$ de Fraley, Waller y Brennan (2000). El instrumento consta de 36 ítems, que evalúa dos dimensiones, la ansiedad definida como el miedo al rechazo y al abandono, y la variable evitación refiere al malestar que genera la cercanía y dependencia a otros (Fraley, 2005). Cada escala cuenta con 18 ítemes evaluados en una escala Likert de 1 a 7 , donde 1 significa no me representa para nada y 7 me representa completamente. En este estudio la instrucción consideró la evaluación en relación a la madre o la persona que haya operado como cuidador principal, pues uno de los supuestos básicos de la teoría del apego es la transferencia de la relación de apego de él o la cuidadora principal (habitualmente la madre) a las relaciones de pareja (Bradbury, Finchman \& Beach, 2000; Feeney et al., 1986; Pietromonaco \& Feldman-Barret, 2000). A su vez, Fraley (2005) refiere que el cuestionario puede ser empleado para evaluar la relación de apego con distintas personas (e.g. padre, madre, amigos, pareja amorosa, cónyuge) sólo modificando las instrucciones originales. Se eliminaron los ítems 9,10 y 11 de la escala de ansiedad, pues tenían una baja carga en ambos factores. El instrumento quedó constituido por 33 ítems, 15 en la escala de ansiedad y 18 en la escala de evitación. La confiabilidad de la escala total medida a través del alfa de Cronbach fue de .90 , y para las escalas de ansiedad y evitación, de .88 y .92 respectivamente.

Evaluación de la sintomatología depresiva. Se empleó la escala Center for Epidemiologic Studies Depression Scale (CES-D) de Radloff (1977). Este es un cuestionario de 20 ítems que tiene como objetivo medir sentimientos y comportamientos depresivos, evaluados según su frecuencia de aparición durante la semana pasada a la administración del test. La consistencia interna de la escala medida a través del alfa de Cronbach fue de .87. Como punto de corte entre ausencia y presencia de síntomas depresivos, se empleó el puntaje 24 establecido por Gempp, Avendaño y Muñoz (2004) para jóvenes chilenos.

\section{Estrategia de análisis}

Primeramente se realizó un análisis de confiabilidad y validez de los instrumentos, para posteriormente realizar un análisis descriptivo de las variables, considerando las diferencias de género. Posteriormente se describieron las características de la relación, en términos del tipo de relación establecida y duración. Finalmente se realizó un análisis de regresión múltiple para evaluar el peso de las variables en la satisfacción con la relación.

\section{Resultados}

\section{Estadísticos descriptivos}

En la Tabla 1, se observan las medias, desviaciones típicas y las diferencias de género entre las variables. Utilizando la prueba $t$ para muestras independientes, se observa la existencia de diferencias de género sólo en la variable depresión $t(118)=3.08, \mathrm{p}<.01$, obteniendo las mujeres puntajes más altos $(M=18.80)$ que los varones $(M=13.54)$. El $27.0 \%$ de las mujeres y el $7.0 \%$ de los varones presentaron síntomas depresivos.

Tabla 1

Medias, Desviaciones típicas y prueba t por género

\begin{tabular}{lccc}
\hline Variables & $\mathrm{M}$ & $\mathrm{DT}$ & $\mathrm{t}(118)$ \\
\hline Satisfacción relacional & 26.03 & 3.98 & -.50 \\
Ansiedad & 2.19 & 1.09 & .65 \\
Evitación & 2.93 & 1.26 & -.46 \\
Depresión & 16.30 & 9.65 & $3.08^{* *}$ \\
Miedo a la intimidad & 70.15 & 16.21 & -.10 \\
\hline${ }^{* *} p<.01$ & & &
\end{tabular}

A su vez, en la Tabla 2 se observa que todas las variables se encuentran relacionadas significativamente entre sí, a excepción de las variables depresión y evitación, y entre ésta última y la satisfacción relacional.

Tabla 2

Correlaciones entre las variables

\begin{tabular}{llllll}
\hline Variables & 1 & 2 & 3 & 4 & 5 \\
\hline $\begin{array}{l}\text { Satisfacción } \\
\text { relacional }\end{array}$ & 1 & & & & \\
Ansiedad & $-.28^{* *}$ & 1 & & & \\
Evitación & -.16 & $.30^{* *}$ & 1 & & \\
$\begin{array}{l}\text { Depresión } \\
\text { Miedo a la }\end{array}$ & $-.23^{* *}$ & $.35^{* *}$ & .02 & 1 & \\
intimidad & $-.48^{* *}$ & $.22^{*}$ & $.29^{* *}$ & $.24^{* *}$ & 1 \\
\hline$* *$ & $p=.01$ (bilateral) * $p=.05$ (bilateral) & & &
\end{tabular}




\section{Características de las relaciones de pareja}

Respecto del tipo de relación que establecían los jóvenes, se constató que el $79.2 \%$ de ellos se encontraban en una relación de pololeo, siendo este tipo de relación predominante en todos los tramos de edad. Las relaciones de menor compromiso fueron más frecuentes entre los más jóvenes, tal como se muestra en la Tabla 3.

Tabla 3

Tipo de relación de pareja por tramo de edad en porcentaje

\begin{tabular}{lcccrcc}
\hline \multirow{2}{*}{ Edad } & \multicolumn{5}{c}{ Tipo de relación de pareja } \\
& saliendo & pololeando & novios & convivencia & otro & Porcentaje \\
\hline $17-19$ & 20.9 & 72.1 & 4.7 & - & 2.3 & 100 \\
$20-22$ & 7.0 & 89.5 & - & - & 3.5 & 100 \\
$23-26$ & 5.0 & 65.0 & 10.0 & 15.0 & 5.0 & 100 \\
Total & 11.7 & 79.2 & 3.3 & 2.5 & 3.3 & 100 \\
\hline
\end{tabular}

Mediante un análisis de ANOVA y utilizando la prueba de Tukey para establecer diferencias intergrupales, se observó que el grado de satisfacción con la relación de pareja, varió dependiendo del tipo de relación $F(4)=9.45$ $\mathrm{p}<.001$, estando más satisfechos los jóvenes con relaciones de pareja más estables, que los que se encontraban en una relación de conocimiento inicial, tal como se observa en la Tabla 4. No se encontraron diferencias significativas en la satisfacción con la relación según la duración de éstas $F(5)=2.02, \mathrm{p}>.05$.

Tabla 4

Tipo de relación y satisfacción

\begin{tabular}{ll}
\hline Tipo de relación & \\
\hline Saliendo & $3.21 \mathrm{a}$ \\
Pololeando & $4.19 \mathrm{~b}$ \\
Novios & $4.61 \mathrm{~b}$ \\
Matrimonio/convivencia & $4.71 \mathrm{~b}$ \\
Otro & $3.52 \mathrm{ab}$ \\
\hline
\end{tabular}

Nota. Los promedios en la misma columna que no comparten el suscriptor difieren en $\mathrm{p}<.05$ en la comparación de grupos de Tukey.

La duración de las relaciones fue variable. Obtuvieron mayor representación porcentual aquellas relaciones cuya duración era de menos de dos meses, y entre uno a dos años. Aquellas relaciones que se extendían por más de dos años representaron aproximadamente un tercio de las relaciones (tabla 5).
Tabla 5

Extensión de la relación en porcentaje por meses y años

\begin{tabular}{lc}
\hline Tiempo de relación & Porcentaje \\
\hline menos de 2 meses & 20.2 \\
2 - 6 meses & 8.4 \\
7 - 11 meses & 11.8 \\
1 año - 1 año 11 meses & 28.6 \\
2 - 3 años & 15.1 \\
3 años y más & 16.0 \\
Total & 100.0 \\
\hline
\end{tabular}

\section{Satisfacción con la relación, apego, depresión e} intimidad

Para evaluar el peso de las variables evitación, ansiedad, depresión y miedo a la intimidad, en la satisfacción relacional, se realizó un análisis de regresión múltiple, empleando el método de pasos sucesivos. La ecuación incluyó una constante de 35.08 , con un coeficiente estandarizado de $\beta=-.442$ para el miedo a la intimidad y de $\beta=-.181$ para la ansiedad. El miedo a la intimidad tiene un mayor peso en la determinación de la satisfacción con la relación, que la ansiedad.

\section{Discusión}

Las experiencias de los jóvenes en relaciones de pareja son predominantemente satisfactorias, siendo mayor en el caso de las relaciones de mayor estabilidad y compromiso, 
independientemente del tiempo involucradas en ellas, lo cual es esperable en este período evolutivo (Arnett, 2000). A su vez, las relaciones de mayor compromiso y estabilidad tienden a darse más frecuentemente, a medida que los jóvenes se acercan al término de la adultez emergente, asemejándose a las relaciones de pareja adultas.

Las variables que determinan la satisfacción con la relación refieren al grado de ansiedad que experimentan los jóvenes ante el temor a perder al otro, lo que se asocia con estilos de apego inseguros, ya sea en la línea de los apegos preocupados o temerosos, que se caracterizan por presentar altos montos de ansiedad y por lo mismo, tienden a buscar la cercanía en las relaciones de pareja y mucho temor a su término (Brennan et al., 1998). A su vez, el miedo a la intimidad es otra de las variables que disminuyen la satisfacción con la relación, dimensión que constituye una de las tareas del desarrollo emocional de los jóvenes.

El hecho de que en este estudio las variables que determinan la satisfacción con la relación de pareja se vinculen con los montos de ansiedad y el miedo a la intimidad, es coherente, pues aún cuando estas variables son diferentes, presentan una relación significativa entre sí. Lo que llama la atención es que la variable evitación no fuese un determinante significativo de la satisfacción relacional, pues otros estudios sostienen lo contrario y que determina a su vez, las características de la relación (Collins, Cooper \& Allard, 2002). Hipotéticamente esto podría vincularse con que no se dé una transferencia de la relación de apego con el cuidador primario, a las relaciones de pareja en forma directa y consistente. Furman, Simon, Shaffer y Bouchey (2002) refieren que la relación entre los estilos de apego con el cuidador primario y el estilo de apego en las relaciones amorosas y de amistad son distintas, pero se encuentran vinculadas entre sí. De hecho, refieren que los modelos operativos en las relaciones de amistad, se encuentran vinculados con los modelos operativos con los padres y los modelos operativos en las relaciones de pareja. Sin embargo, los modelos operativos con los padres y aquellos en las relaciones de pareja, se encuentran inconsistentemente vinculados. Esto podría estar avalando la existencia de modelos operativos específicos a las relaciones con los padres, amistades y parejas (Collins et al., 1994). También estos hallazgos pueden indicar diferencias culturales, pues los montos de ansiedad y evitación varían en diferentes contextos socioculturales (Schmitt et al., 2003), siendo necesario que futuras investigaciones profundicen en este aspecto, considerando a su vez la especificidad de la relación que se desea evaluar.

No se observaron diferencias de género en el miedo a la intimidad, lo cual no es concordante con otras investigaciones (Taylor et al., 2000), donde se constata que las mujeres valoran en mayor medida que los varones las relaciones de cercanía, autoexposición y expresión de sentimientos amorosos. De aquí surge la relevancia de investigar los cambios socioculturales y de género en la adultez emergente. La mujeres presentan más depresión que los varones, lo cual es concordante con estudios epidemiológicos (MINSAL, 2004). Sin embargo, la depresión, ni en los hombres ni en mujeres afecta su satisfacción con la relación, lo cual se puede deber a que los síntomas que presentan en su mayoría no son de alta gravedad.

Dentro de las limitaciones del estudio se constata la dificultad en definir el objeto de estudio, dada la variabilidad de las significaciones que puede asumir una relación amorosa. A su vez, es necesario revisar con más profundidad las características psicométricas del instrumento utilizado para la evaluación de la ansiedad y evitación, en muestras distintas a las anglosajonas y considerar la evaluación del apego en base a la relación que en particular se desea evaluar, tal como lo recomiendan Crowell, Fraley y Shaver (1999). Así se puede establecer con mayor precisión las asociaciones entre ellas para profundizar el conocimiento de la transferencia de los modelos operativos internos a distintas relaciones.

\section{Referencias}

Arnett, J. (1998). Learning to stand alone: The contemporary American transition to adulthood in cultural and historical context. Human Development, 41, 295-315.

Arnett, J. (2000). Emerging adulthood: A theory of development from the late teens through the twenties. American Psychologist, 55, 469480 .

Arnett, J. (2001). Conceptions of the transition to adulthood: Perspectives from adolescence to midlife. Journal of Adult Development, 8 , 133-143.

Bartholomew, K., \& Horowitz, L. (1991). Attachment style among young adults: a test of four-category model. Journal of Personality and Social Psychology, 61, 226-244.

Bowlby, J. (1988). A secure base: Clinical applications of attachment theory. London: Routledge.

Bradbury, T., Fincham, F., \& Beach, S. (2000). Research on the nature and determinants of marital satisfaction: A decade in review. Journal of Marriage and the Family, 62, 964-980.

Brennan, K., Clark, C., \& Shaver, P. (1998) Self-report measurement of adult attachment: An integrative overview. En: J. A. Simpson \& W. S. Rholes (Eds.), Attachment Theory and Close Relationships (46-76). New York: Guilford Press.

Brumbaugh, C. C., \& Fraley, R. C. (2006). Transference and attachment: How do attachment patterns get carried forward from one relationship to the next? Personality and Social Psychology Bulletin, 32, 552-560.

Cassidy, J. (2001). Truth, lies, and intimacy: An attachment perspective. Attachment and Human Development, 3, 121-155.

Cohen, P., Kasen, S., Chen, H., Hartmark, C., \& Gordon, K. (2003). Variations in patterns of developmental transitions in the emerging adulthood period. Developmental Psychology, 39, 657-669.

Collins, W. (2003). More than myth: The developmental significance of romantic relationships during adolescence. Journal of Research on Adolescence, 13, 1-24.

Collins, N., Cooper, L., \& Allard, L. (2002). Psychosocial vulnerability from adolescence to adulthood: A prospective study of attachment style differences in relationship functioning and partner choice. Journal of Personality, 70, 964-1006.

Collins, N. L., Guichard, A. C., Ford, M. B., \& Feeney, B. C. (2004). Working models of attachment: New developments and emerging themes. En: W. S. Rholes \& J. A. Simpson (Eds.) Adult Attachment: Theory, Research, and Clinical Implications (196-239). New York: Guilford. 
Collins, N. L., \& Read, S. J. (1994).Cognitive representation of attachments: The structure and function of working models. En: K. Bartholomew \& D. Perlman (Eds.), Advances in Personal Relationships: Vol. 5. Attachment processes in adulthood (53-90). London: Jessica Kingsley.

Collins, W. A., \& Sroufe, L. A. (1999). Capacity for intimate relationships: A developmental construction. En: W. Furman, B. B. Brown \& C. Feiring (Eds.), The Development of Romantic Relationships in Adolescence (125-147). Cambridge, England: Cambridge University Press .

Connolly, J., \& Goldberg, A. (1999). Romantic relationship in adolescence: the role of friends and peers in their emergence and development. En: W. Furman et al., (Eds.), The Development of Romantic Relationships in Adolescence (266-290). New York: Cambridge University Press.

Crowell, J., Fraley, C., \& Shaver, P. (1999). Measurement of individual differences in adolescent and adult attachment. En: J. Cassidy \& P. Shaver. (Eds.), Handbook of attachment theory and research (434-465). New York: Guilford Press.

Dávila, J., Steinberg, S.J., Kachadourian, L., Cobb, R., \& Fincham, F. (2004). Romantic involvement and depressive symptoms in early and late adolescence: The role of preoccupied relational style. Personal Relationships, 11, 161-178.

Descoutner, C., \& Thelen, M. (1991). Development and validation of a Fear-of-Intimacy Scale. Psychological Assessment: A Journal of Consulting and Clinical Psychology, 3, 218-225.

Dottan, R. F., Cohen, O., Iwaniec, D., Sapir, Y., \& Weizman, A. (2003). The drug-user husband and his wife: Attachment styles, family cohesion and adaptability. Journal of Substance Use and Misuse, 38, 271-292.

Erikson, E. H. (1963). Childhood and society. ( $2^{\text {nd }}$ ed.). New York: Norton Press.

Feeney, J. \& Noller, P. (1986). Adult Attachment. London: Sage

Ferrer, V., Bosch, E., Navarro, C., Ramis, M., \& García, E. (2008). El concepto del amor en España. Psicothema, 20, 589-595.

Fraley, C. (2005). Information on the Experiences in Close RelationshipsRevised (ECR-R) Adult Attachment Questionnaire. Recuperado en Noviembre 20, 2009, de en http://www.psych.uiuc.edu/ rcfraley/ measures/ecrr.htm

Fraley, C., Waller, N. \& Brennan, K. (2000). An item response theory analysis of self-report measure of adult attachment. Journal of Personality and Social Psychology, 78, 350-365.

Furman, W., Simon, V., Shaffer, L., \& Bouchey, H. (2002). Adolescents' working models and styles for relationships with parents, friends, and romantic partners. Child Development, 73, 241-255.

Gaudet, S. (2007). Emerging Adulthood: A New Stage in the Life Course. Implications for Policy Development. Goberment of Canada. Recuperado en agosto 19, 2009, disponible en http-www.policyresearch. gc.ca-dodiclib-DP_YOUTH_Gaudet_200712_e.url.

Gempp, R., Avendaño, C., \& Muñoz, C. (2004). Normas y punto de corte para la Escala de Depresión del Centro para Estudios Epidemiológicos
(CES-D) en población juvenil chilena. Revista Terapia Psicológica, $22,145-156$.

Hazan, C. \& Shaver, P. (1987). Romantic love conceptualized as an attachment process. Journal of Personality and Social Psychology, $52,511-524$.

Hendrick, S. (1988). A generic measurement of relationship satisfaction. Journal of Marriage and the Family, 50, 93-98.

Instituto Nacional de la Juventud (2006). 5ta Encuesta Nacional de Juventud. Chile: Instituto Nacional de la Juventud. Gobierno de Chile.

Kirkpatrick, L.A., \& Davis, K.E. (1994). Attachment style, gender, and relationship stability: A longitudinal analysis. Journal of Personality and Social Psychology, 66, 502-512.

Kobak, R. R., Sudler, N., \& Gamble, W. (1991). Attachment and depressive symptoms during adolescence: A developmental pathways analysis. Development and Psychopathology, 3, 461-474.

Marchand-Reilly, J. (2009). Depressive symptoms in young adults: The role of attachment orientation and romantic relationship conflict. Journal of Adult Development, 16, 31-38.

McNally, A. M., Palfai, T. P., Levine, R. V., \& Moore, B. M. (2003). Attachment dimensions and drinking-related problems among young adults. The mediational role of coping motives. Addictive Behaviors, 28, 1115-1127.

Ministerio de Salud (2004). Encuesta Nacional de Salud Chile 2003. Recuperado en Octubre 14, 2009, de http--escuela.med.puc.cl-deptossaludpublica-ResultadoENS-CapIV209Depresion.pdf

Pietromonaco, P., \& Feldman-Barrett, L. (2000a). The internal working models concept: What do we really know about the Self in relation to Others? Review of General Psychology, 4, 155-175.

Radloff, L. S. (1977). The CES-D Scale: A self-report depression scale for research in the general population. Applied Psychological Measurement, $1,385-401$.

Sánchez, V., Ortega, F.J., Ortega, R., \& Viejo, C. (2008). Las relaciones sentimentales en la adolescencia: Satisfacción, conflictos y violencia. Escritos de Psicología, 2, 97-109.

Schmitt, D., et al., (2003). Are men universally more dismissing than women? Gender differences in romantic attachment across 62 cultural regions. Personal Relationships, 10, 307-331.

Shulman, S., \& Scharf, M. (2000). Adolescent romantic behaviors and perceptions: age-related differences and links with family and peer relationships. Journal of Research on Adolescence, 10, 99-118.

Shulman, S., \& Seiffge-Krenke, I. (2001). Adolescent romance: between experience and relationships. Journal of Adolescence, 24, 417-428.

Taylor, S.E., Klein, L. C., Lewisa, B.P., Gruenewald, T.L., Gurung, R.A., \& Updegraff, J.A. (2000). Biobehavioral responses to stress in females: Tend-and-befriend, not fight-or-flight. Psychological Review, 107, 411-429. 